$\begin{array}{cc}\text { ACADEMIA ROMÂNĂ } & \text { Rev. Roum. Chim., } \\ \text { 2021, 66(2), 205-209 }\end{array}$

Note

\title{
SYNTHESIS AND CHARACTERIZATION OF A NEW HYDRAZYL FREE RADICAL, A FORMYL-DERIVATIVE OF DPPH
}

\author{
Petre IONITA
}

University of Bucharest, Faculty of Chemistry, 90-92 Panduri, Bucharest, Roumania

Starting from 2,2-diphenyl-1-(2,4,6-trinitropheyl)hydrazine and urotropine in the presence of trifluoracetic acid it was obtained a formyl derivative as a yellow solid, that can form under oxidative condition the persistent hydrazyl free radical 2-(p-formyl-phenyl)-2-phenyl-1-(2,4,6trinitrophenyl)hydrazyl having a violet colour. The same formyl hydrazine derivative in the presence of a base forms the corresponding anion with a red-brown colour. The new compounds were characterized by appropriate means, like NMR, IR, UV-VIS, ESR.

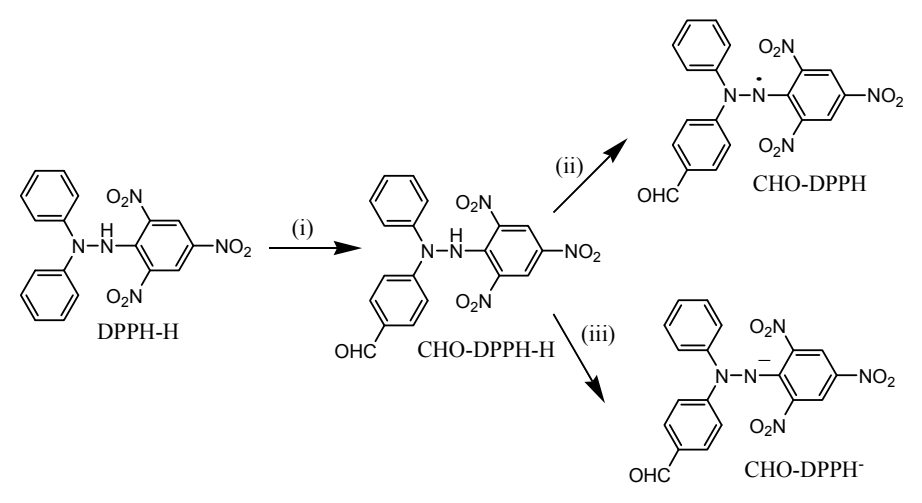

\section{INTRODUCTION}

Hydrazyl free radicals are an interesting topic in chemistry, since the discovery, about 100 years ago, of the so called DPPH free radical (2,2diphenyl-1-(2,4,6-trinitropheyl)hydrazyl). ${ }^{1}$ Meantime, many other derivatives and congeners were synthesized and their properties studied. ${ }^{2-5}$ Their stability was a subject of high debate, and it can be mentioned the pioneering work of Prof. Balaban and collaborators that pinpoint the factors affecting the stability and equilibria of such nitrogen centered free radicals. ${ }^{6,7}$
Some $p$-phenyl derivatives of DPPH are known, containing methyl-, hydroxyl-, bromo-, or nitrogroups (Figure 1). ${ }^{8,9}$ The substitution of a $\mathrm{H}$-atom from the $p$-phenyl position usually induce a strong change in the physical and chemical behavior. For example, addition a nitro-group in the $p$-phenyl position of DPPH changes the following: in visible spectroscopy, the absorption wavelength from about 524 to $495 \mathrm{~nm}$; in ESR, the hyperfine coupling constants from almost equivalent nitrogen atoms with hyperfine coupling constants $\mathrm{a}_{\mathrm{N} 1}=\mathrm{a}_{\mathrm{N} 2} \sim 9 \mathrm{G}$ to about $\mathrm{a}_{\mathrm{N} 1}=10.43 \mathrm{G}$ and ${ }_{\mathrm{aN} 2}=6.95 \mathrm{G} ;{ }^{8,10}$ in the case of the reduced counterpart of DPPH, usually denoted as $\mathrm{DPPH}-\mathrm{H}$, the acidity constant of the $\mathrm{NH}$ group is lowered from 8.54 to $7.35 p \mathrm{~K}_{\mathrm{a}}$ units. $^{11}$

\footnotetext{
*Corresponding author: p_ionita@yahoo.co.uk
} 


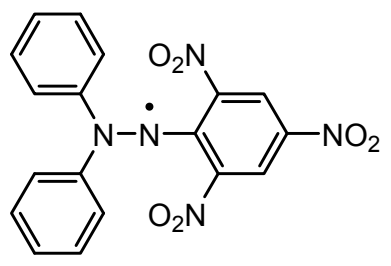

DPPH

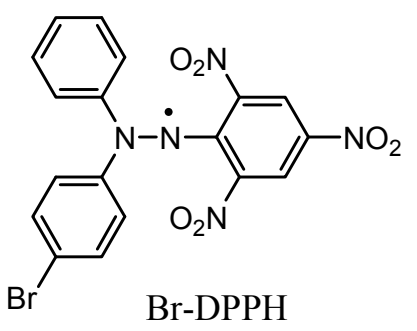

Br-DPPH

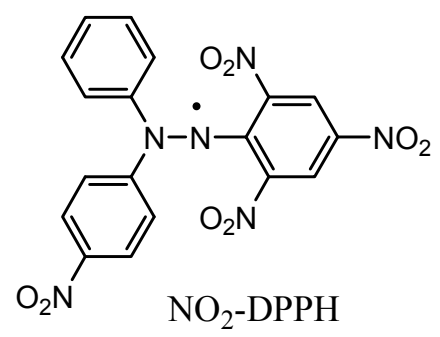

Fig. 1 - Structure of DPPH and some $p$-phenyl derivatives.

In the quest of finding new hydrazyl stable free radicals with interesting properties that can be pursued in novel applications, ${ }^{12}$ this work deals with the synthesis and characterization of a new compound derived from DPPH, in which a formyl group was inserted into the para-position of a phenyl ring.

\section{RESULTS AND DISCUSSION}

The synthesis of $p$-phenyl derivatives of DPPH usually follows two routes. ${ }^{8}$ In the first one, the starting material is a substituted $p$-diphenyl amine, that undergoes reaction of nitrosation, reduction to the corresponding hydrazine and coupling with picryl chloride (1-chloro-2,4,6-trinitrobenzene); the picryl-hydrazine thus obtained is further oxidized to the hydrazyl free radical using solid potassium permanganate, lead dioxide or silver oxide. For the second route, DPPH or its reduced counterpart DPPH-H reacts in a radical reaction with specific regents, like bromine or nitrogen dioxide and forms directly the corresponding $p$-substituted derivative. ${ }^{2,3,9,13}$ Although the second way seems simpler, it can be used only for these specific reagents that are or can form free radicals. As an example, $p$-nitrophenyl derivative of DPPH (usually denoted in literature as $\left.\mathrm{NO}_{2}-\mathrm{DPPH}\right)$ cannot be obtained using the first route, as nitro-group will be reduced during synthesis steps; however, this compound is selectively obtained through a radical + radical coupling, from DPPH and $\mathrm{NO}_{2} .{ }^{10}$

Introduction of a formyl group can be achieved is a single step for aromatic compounds using urotropine (hexamethylenetetramine) and trifluoroacetic acid. ${ }^{14}$ This procedure was found to work in the case of DPPH-H, while another classical one (DMF and $\left.\mathrm{POCl}_{3}\right)^{15}$ didn't work. Therefore, starting from DPPH-H the formylderivative CHO-DPPH-H (Figure 2) was obtained. The compound of interest was separated from a complex mixture using column chromatography or preparative TLC (see Experimental).

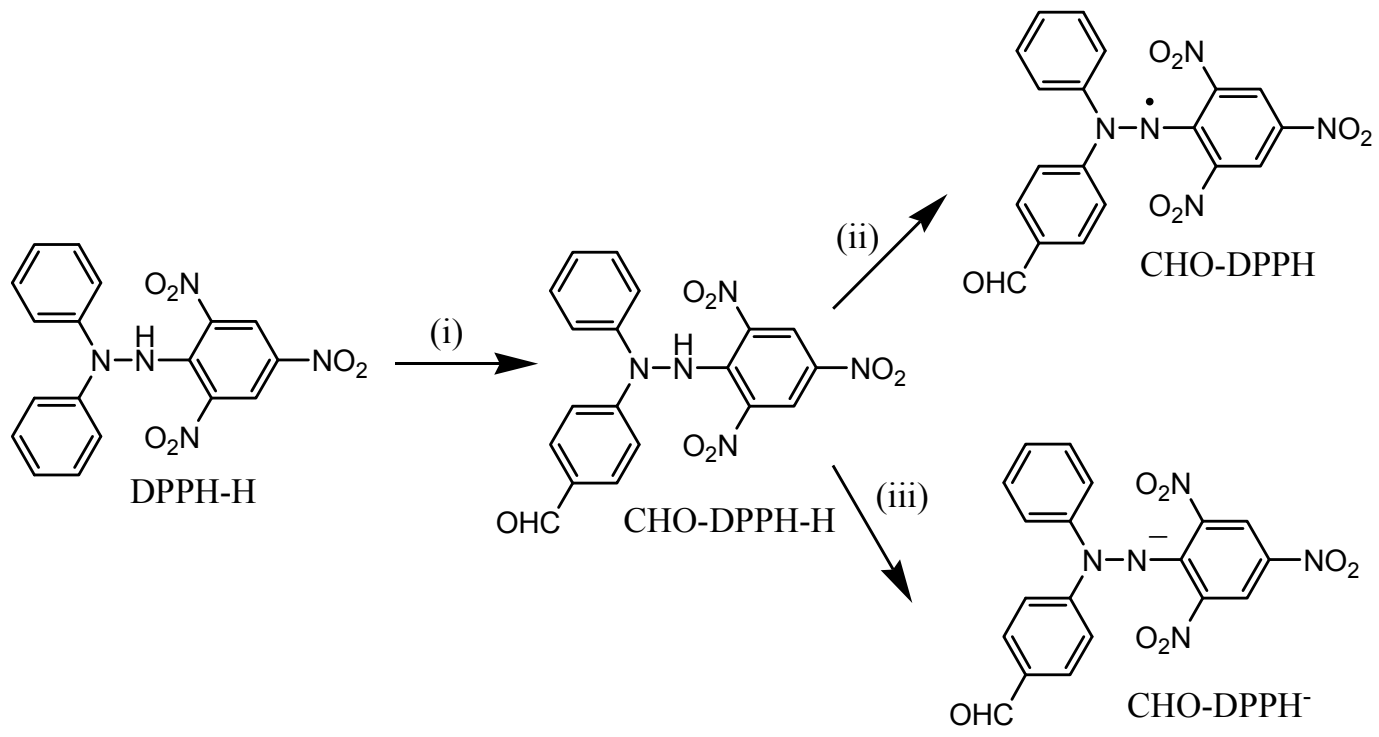

Fig. 2 - Synthesis of CHO-DPPH: i) urotropine/TFA; ii) $\mathrm{PbO}_{2}$; iii) $\mathrm{KOH}$. 


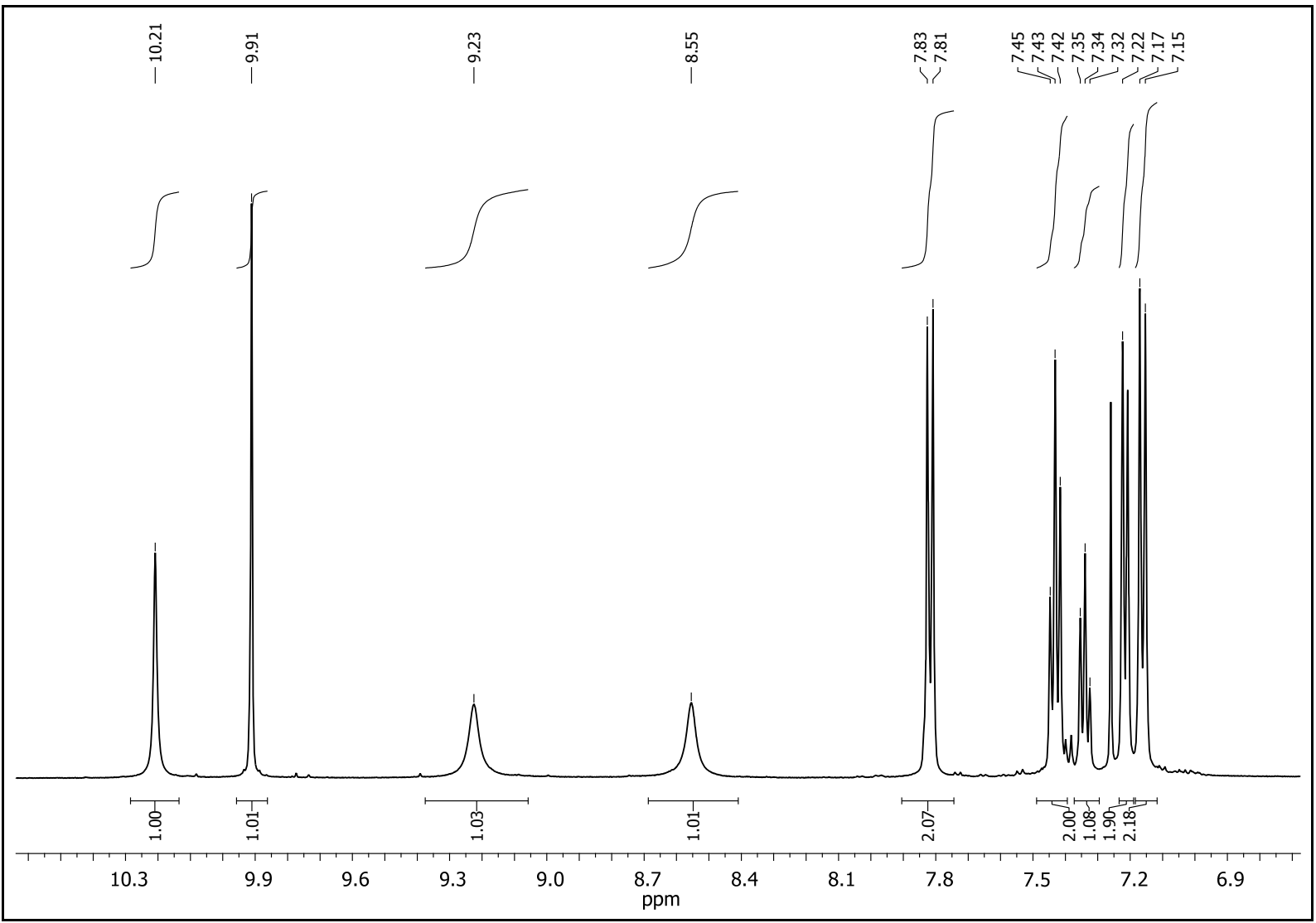

Fig. $3-{ }^{1} \mathrm{H}-\mathrm{NMR}$ spectrum of CHO-DPPH-H in $\mathrm{CDCl}_{3}$.

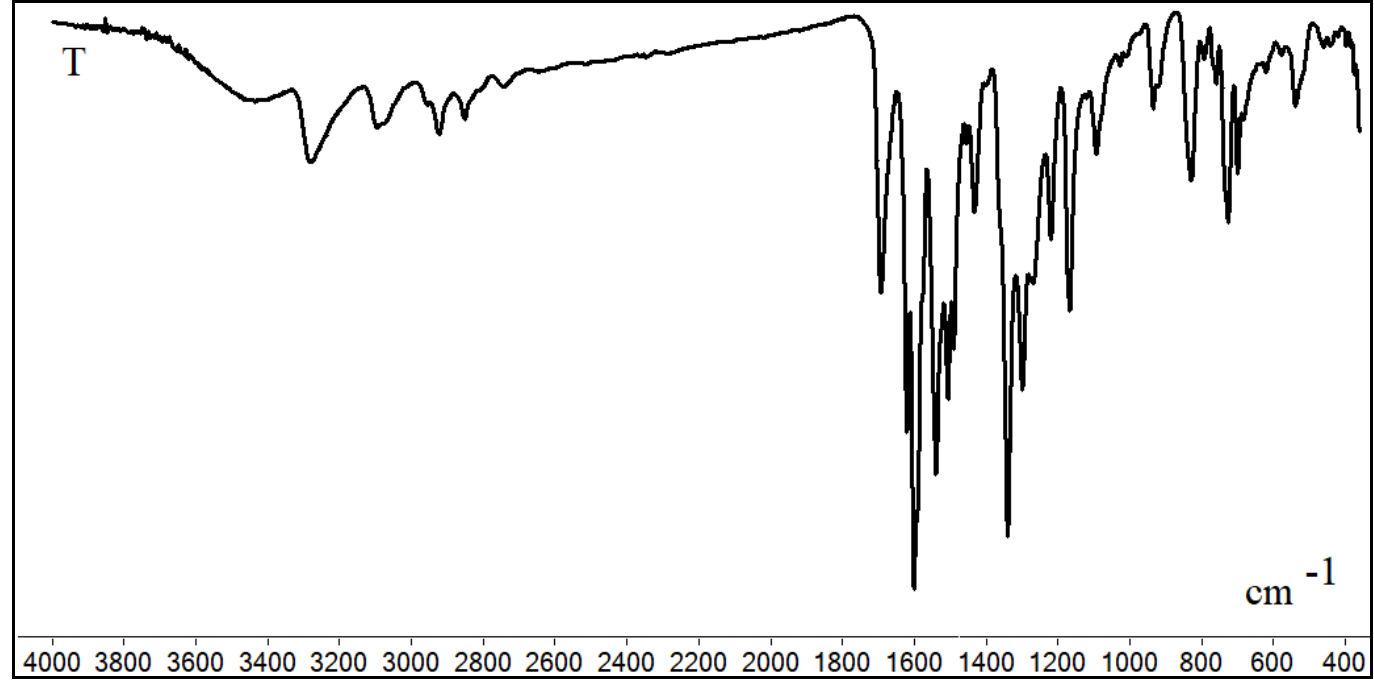

Fig. 4 - IR spectrum of CHO-DPPH-H.

Characterization of the CHO-DPPH-H was performed using several techniques. Thus, in the ${ }^{1} \mathrm{H}-\mathrm{NMR}$ spectrum (Figure 3 ) there is noticed the $\mathrm{NH}$ proton at $10.21 \mathrm{ppm}$, followed by the $\mathrm{CHO}$ one at $9.91 \mathrm{ppm}$. The two $\mathrm{CH}$ from the picryl moiety appear as different singlets at $9.23 \mathrm{ppm}$ and $8.55 \mathrm{ppm}$. The $H$-nuclei from the two phenyls ring are located between 7.9-7.1 ppm, with the corresponding splitting. In the ${ }^{13} \mathrm{C}-\mathrm{NMR}$ spectrum it is necessary to mention the $\mathrm{CHO}$ peak at 190.63 ppm, thus confirming the proposed structure.

In the IR spectrum (Figure 4), the most intense band is noticed at $1601 \mathrm{~cm}^{-1}$, being due to the formyl group; other groups are present as amino (3279 $\left.\mathrm{cm}^{-1}\right)$, nitro $\left(1339\right.$ and $\left.1539 \mathrm{~cm}^{-1}\right)$, and aromatic rings $\left(3094 \mathrm{~cm}^{-1}\right)$, supporting the proposed structure. 


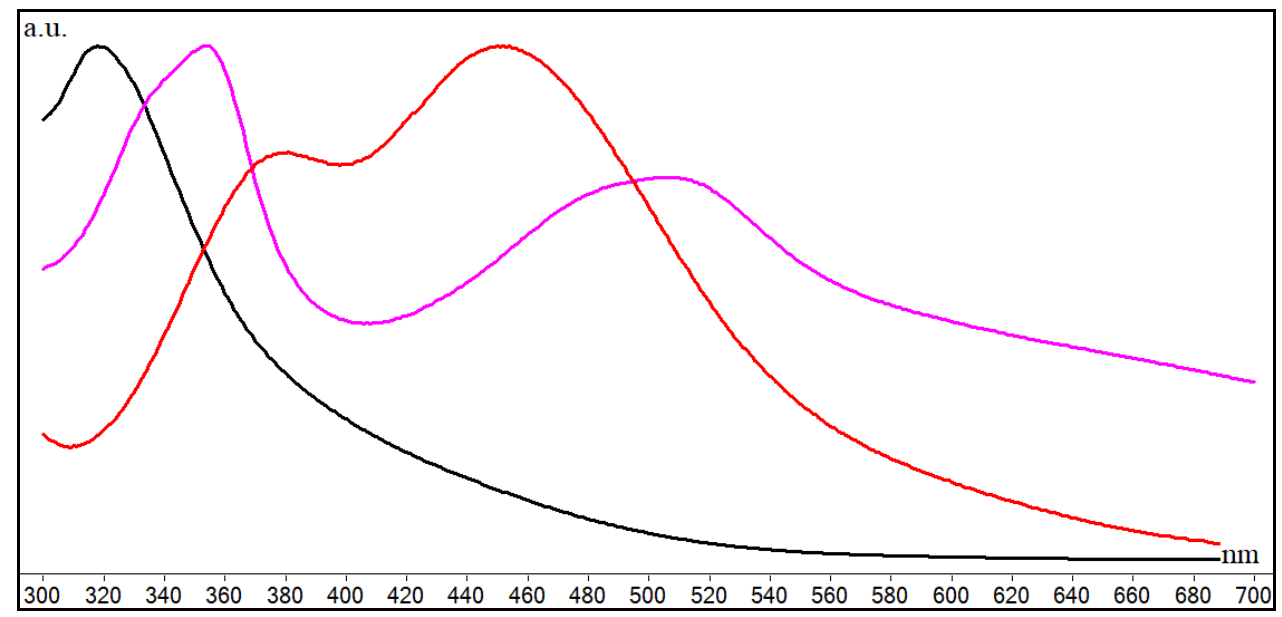

Fig. 5 - UV-VIS spectra of CHO-DPPH-H hydrazine (black), CHO-DPPH free radical (violet) and the corresponding anion $\mathrm{CHO}_{-} \mathrm{DPPH}^{-}($red).

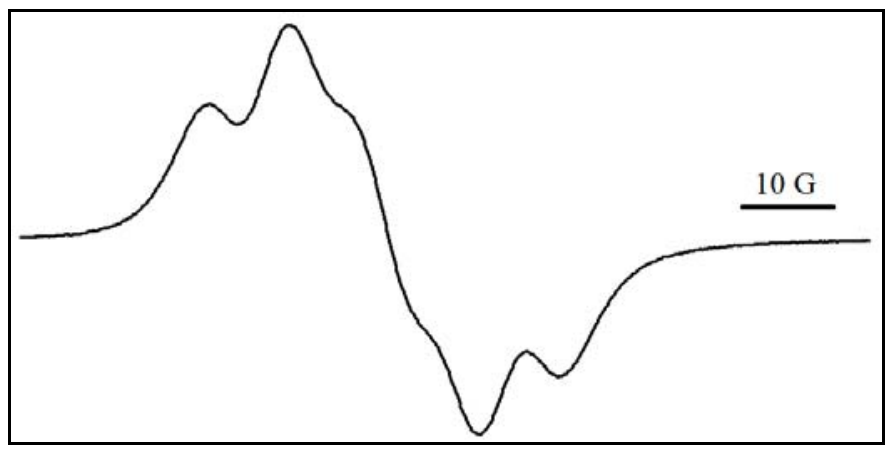

Fig. 6 - ESR spectrum of CHO-DPPH free radical.<smiles>O=Cc1ccc(N(c2ccccc2)N(c2ccccc2)c2c([N+](=O)[O-])cc([N+](=O)[O-])cc2[N+](=O)[O-])cc1</smiles>

Fig. 7 - Over-oxidation of CHO-DPPH free radical.

An interesting and well-known property of such derivative is their colour change, process that can be simply followed by UV-Vis (Figure 5). Thus, the hydrazine CHO-DPPH-H has a $1_{\max }=318 \mathrm{~nm}$, being yellow in colour, while the free radical CHO-DPPH has a $1_{\max }=506 \mathrm{~nm}$ and is violet; the corresponding anion $\mathrm{CHO}-\mathrm{DPPH}^{-}$has a $1_{\max }=$ $451 \mathrm{~nm}$, with a red-brown colour.

ESR spectrum shown in Figure 6 is that one undoubtedly confirming the free radical structure of CHO-DPPH. The measured hyperfine coupling constants are about $\mathrm{a}_{\mathrm{N} 1}=10.5 \mathrm{G}$ and $\mathrm{a}_{\mathrm{N} 2}=8.5 \mathrm{G}$. Regarding the shape of the spectrum, it can be observed five peaks with large linewidth, commonly recorded for DPPH derivatives.
Although the CHO-DPPH free radical can be regarded as a stable or persistent hydrazyl type free radical, an interesting behaviour was observed checking by TLC the progress of the oxidation reaction from CHO-DPPH-H to CHO-DPPH (oxidation performed in DCM with lead dioxide). It was found that in time the CHO-DPPH free radical undergoes an interesting transformation, yielding the previously known quinonoid derivative (Figure 7). ${ }^{9}$

At this time the mechanism for this transformation (Figure 7) is not clear and further investigations are necessary. It can be only supposed that $p$-formyl-group is also oxidized in a first instance to a carbonyl radical that undergoes 
stabilization by pairing with the free hydrazyl electron altogether with $\mathrm{CO}$ or $\mathrm{CO}_{2}$ elimination.

In conclusion, synthesis of novel hydrazyl free radicals with specific properties and containing groups that can be easily converted into other moieties of interest is still an attractive subject in this area. The new derivative of DPPH can be further be involved in many other organic processes of interest due to the high reactivity of formyl group.

\section{EXPERIMENTAL}

All chemicals and materials, including solvents, were purchased from Merck or Chimopar. NMR spectra were recorded in $\mathrm{CDCl}_{3}$ on a Bruker Avance III $500 \mathrm{MHz}$. IR spectrum was recorded on a FT-IR Bruker Vertex 70 spectrometer. UV-Vis spectra were recorded in methanol on a double-bean UVD-3500 spectrometer. ESR spectrum was recorded in toluene on a JES-FA 100 apparatus.

Synthesis of $\mathrm{CHO}-\mathrm{DPPH}-\mathrm{H}, \quad$ 2-(p-formyl-phenyl)-2phenyl-1-(2,4,6-trinitrophenyl)hydrazine or 4-(1-phenyl-2(2,4,6-trinitrophenyl)hydrazynyl)benzaldehyde, $\mathrm{C}_{19} \mathrm{H}_{13} \mathrm{~N}_{5} \mathrm{O}_{7}$. $200 \mathrm{mg}$ DPPH-H $(0.5 \mathrm{mmol})$ and $560 \mathrm{mg}$ urotropine $(4 \mathrm{mmol})$ were suspended in about $2 \mathrm{~mL}$ DCM to which under stirring was added dropwise $2 \mathrm{~mL}$ trifluoroacetic acetic and the mixture left overnight. Next day were added about $50 \mathrm{~mL}$ DCM and $50 \mathrm{~mL}$ of water and under vigorous stirring was added also solid sodium hydrogen carbonate till the aqueous solution reach $\mathrm{pH} 8$. The organic phase was separated, dried over anhydrous sodium sulphate and the solvent removed using a rotavap. The residue was chromatographied on silica gel using DCM as eluent. Yields $35 \% . \mathrm{R}_{\mathrm{f}} 0.50(\mathrm{DCM} / \mathrm{silica}$ gel). $1_{\max }=318 \mathrm{~nm}$ (ethanol); in the presence of $\mathrm{KOH} 1_{\max }=$ $451 \mathrm{~nm}(1 \mathrm{mg}$ compound dissolved in $10 \mathrm{~mL}$ methanolic solution of $\mathrm{KOH}\left(1 \mathrm{mg} \mathrm{KOH} / \mathrm{mL}\right.$ methanol)). ${ }^{1} \mathrm{H}-\mathrm{NMR}$ (ppm, $\left.\mathrm{CDCl}_{3}\right): 10.21(\mathrm{~s}, 1 \mathrm{H}, \mathrm{NH}), 9.91(\mathrm{~s}, 1 \mathrm{H}, \mathrm{CHO}), 9.23(\mathrm{~s}, 1 \mathrm{H}$, CH-picryl), 8.55 (s, 1H, CH-picryl), $7.82(\mathrm{~d}, J=8.5 \mathrm{~Hz}, 2 \mathrm{H}$, CH-benzaldehyde), 7.43 (t, $J=7.8 \mathrm{~Hz}, 2 \mathrm{H}, \mathrm{C} H$-phenyl), 7.34 (t, $J=7.4 \mathrm{~Hz}, 1 \mathrm{H}, \mathrm{CH}$-phenyl), $7.21(\mathrm{~d}, J=8 \mathrm{~Hz}, 2 \mathrm{H}, \mathrm{CH}$ phenyl), 7.16 (d, $J=8.6 \mathrm{~Hz}, 2 \mathrm{H}, \mathrm{CH}$-benzaldehyde). ${ }^{13} \mathrm{C}$ NMR (ppm, $\mathrm{CDCl}_{3}$ ): 190.63, 150.98, 144.62, 141.67, 140.11, $137.17,134.23,132.20,132.17,131.09,130.56,130.46$, 128.37, 126,27, 125.09, 124.12, 116.82. IR $\left(\mathrm{cm}^{-1}\right): 3435$;
$3279 ; 3094 ; 2922 ; 1692 ; 1619 ; 1601 ; 1539 ; 1506 ; 1431 ; 1339$; $1298 ; 1166 ; 1092 ; 924 ; 828 ; 726 ; 538$.

The CHO-DPPH persistent free radical was obtained by oxidation of a DCM solution of CHO-DPPH-H (5 mg in $2 \mathrm{~mL}$ solvent) with lead dioxide $(200 \mathrm{mg}) . \mathrm{R}_{\mathrm{f}} 0.58$ (DCM/silica gel). UV-VIS: $1_{\max }=506 \mathrm{~nm}$ (ethanol). ESR (toluene): $\mathrm{a}_{\mathrm{N} 1}=10.5 \mathrm{G}$ and ${ }_{\text {aN2 }}=8.5 \mathrm{G}$. Over-oxidation affords the $p$-quinonoid (betainic) compound described earlier. ${ }^{9}$

Acknowledgement. The author thanks dr. D. Culita for the IR spectrum and dr. G. Ionita for the ESR spectrum.

\section{REFERENCES}

1. S. Goldschmidt and K. Renn, Ber. Dtsch. Chem. Ges., 1922, 55, 628-643.

2. J. A. Weil, K. V. Sane and J. M. Kinkade, J. Phys. Chem., 1961, 65, 710-712.

3. P. F. Currie, J. W. Quail and J. A. Weil, Can. J. Chem., 1980, 58, 723-726.

4. P. Ionita, M. T. Caproiu, C. Luca, T. Constantinescu, H. Caldararu and A. T. Balaban, J. Label. Cpd. Radiopharm., 1998, XLI, 791-799.

5. P. Ionita, M. T. Caproiu and A. T. Balaban, Rev. Roum. Chim., 2000, 45, 935-941.

6. A. T. Balaban, P. T. Frangopol, M. Frangopol and N. Negoita, Tetrahedron, 1967, 23, 4661-4676.

7. A. T. Balaban, P. T. Frangopol, M. Marculescu and J. Bally, Tetrahedron, 1961, 13, 258-267.

8. A. R. Forrester, J. M. Hay and R. H. Thomson, "Organic chemistry of stable free radicals", Academic Press, London, 1968.

9. P. Ionita, F. Tuna, M. Andruh, T. Constantinescu and A. T. Balaban, Aust. J. Chem., 2007, 60, 173-179.

10. C. Luca, P. Ionita, E. Popa and T. Constantinescu, Rev. Roum. Chim., 1998, 43, 753-756.

11. C. Luca, P. Ionita, M. T. Caproiu, H. Caldararu and T. Constantinescu, Rev. Roum. Chim., 1998, 43, 221-224.

12. B. Patrascu, S. Mocanu, A. Coman, A. M. Madalan, C. Popescu, A. Paun, M. Matache and P. Ionita, Int. J. Mol. Sci., 2020, 21, art no. 3559.

13. L. Gille, U. Prosch and R. Stoesser, Radiat. Phys. Chem., 1992, 40, 461-468.

14. W. E. Smith, J. Org. Chem., 1972, 37, 3972-3973.

15. O. Meth-Cohn and S. P. Stanforth, Compr. Org. Synth., 1991, 2, 777-794. 
